and if one causes the other or vice versa, but their responsibility in the development of severe complications of obesity is undoubted. Considered the greater risk of
NAFLD for IR obese children, it might be advisable to perform LU whenever IR-HOMA is pathologic, regardless of the lack of clinical or laboratory sign of NAFLD.

\title{
34 - Evaluation of oxidative stress markers in obese children
}

\author{
P Matusik' , Z Prokopowicz² , B Norek² , M Olszanecka-Glinianowicz ${ }^{3}$ and E Malecka-Tendera ${ }^{1}$ \\ 'Department of Paediatrics, Endocrinology and Diabetes, Medical University of Silesia, Katowice, Poland: ${ }^{2}$ Upper- \\ Silesian Center for Child's Health, Katowice, Poland: ${ }^{3}$ Department of Pathophysiology, Medical University of \\ Silesia, Katowice, Poland
}

Introduction: Obesity in childhood can increase the risk of cardiovascular morbidity and mortality in adulthood. Oxidative stress seems to be involved in the pathophysiology of atherosclerosis, diabetes and cardiovascular complications in obesity. The aim of our study was to evaluate the level of oxidative stress markers in obese children comparing to the lean control group.

Method: Oxidative stress markers (TOS - total oxidative status, TAC - total antioxidative capacity, oxy-LDL), leptin and adiponectin were determined in forty-two obese children and forty healthy controls. Nutritional status by BMI and waist/height ratio calculation and body composition analysis (Tanita BC-418) was assessed in all children.
Results: TAC was significantly $(P<0 \cdot 0001)$ lower, but oxyLDL level was significantly $(P<0 \cdot 05)$ higher in obese than in healthy children. TOC was significantly correlated with fat percentage $(r=0.494 ; P<0.05)$ measured by bioimpedance and with leptin level $(r=0.518 ; P<0 \cdot 01)$ in obese children.

Conclusions: High level of oxy-LDL together with low antioxidant capacity detected in obese children, and significant relation of nutritional status to total oxidative stress indicate imbalance in oxidative/antioxidative status in obese children. This situation can lead to higher risk of atherosclerotic and diabetic complications in the future.

Funding: Research relating to this abstract was funded by Medical University of Silesia Grant No. KNW-1-183/09.

\section{Poster Abstracts: Physical Activity and Fitness} 35 - The relationship between $\mathrm{BMI}$ and balance in
$7-16$-year-old boys

\author{
$S$ Birch and M Duncan
}

Department of Biomolecular and Sports Science, Coventry University, UK

Introduction: Overweight/obesity negatively influences balance and stability in adults. Research has not assessed whether this is the case in children. The aim of the present study was to determine the relationship between BMI and balance performance in a sample of 7-14-yearold boys.

Method: Two hundred and twenty-nine boys aged 7-16 years (mean age 11.4 (SD 2.4) years) participated in the study following ethics approval. Height and mass were determined using a Seca stadiometer and weighing scales (Seca Instruments, Hamburg, Germany) from which BMI was determined and weight status being classified using International Obesity Task Force criteria. Balance was assessed using a computerised stadiometer (MFT S-3 System, Innsbruck, Austria) and from which two measures of balance; balance stability and sensorimotor balance were determined.

Results: Pearson's correlations indicated that higher BMI was associated with poorer balance stability $(r=-0.392, \quad P=0 \cdot 0001)$ and sensorimotor balance $(r=-0 \cdot 296, P=0 \cdot 0001)$.

Analysis of covariance, controlling for age indicated that balance stability, performance was significantly poorer for children who were 'overweight/obese' compared with 'normal weight' children $(F(1,226)=79 \cdot 2, P=0 \cdot 001)$. Likewise, sensorimotor balance was significantly poorer for 'overweight/obese' children $(F(1,226)=11 \cdot 76, P=0 \cdot 001)$ 